\title{
Co-Axial Extrusion of Tubular Tissue Constructs Using A Gelatin/GelMA Blend Bioink
}

Ying Wang, ${ }^{\dagger}$ Ranjith Kumar Kankala, ${ }^{\dagger},+$ Kai Zhu, ${ }^{\S}$ Shi-Bin Wang, ${ }^{\dagger},+$ Yu Shrike Zhang, $\|,{ }^{*}$ Ai-Zheng Chen ${ }^{\dagger,}$, *

${ }^{\dagger}$ Institute of Biomaterials and Tissue Engineering, Huaqiao University, Xiamen 361021, P. R. China

${ }^{*}$ Fujian Provincial Key Laboratory of Biochemical Technology (Huaqiao University), Xiamen 361021, P. R. China

${ }^{\S}$ Department of Cardiac Surgery, Zhongshan Hospital, Fudan University, Shanghai 200032, P. R. China

"Division of Engineering in Medicine, Department of Medicine, Brigham and Women's Hospital, Harvard Medical School, Cambridge, MA 02139, USA

*Corresponding authors:

Email addresses: yszhang@research.bwh.harvard.edu (Y. S. Zhang) azchen@hqu.edu.cn (A. Z. Chen)

Number of pages: 3 pages

Number of Figures: 2 figures

Number of Tables: 1 table 
A

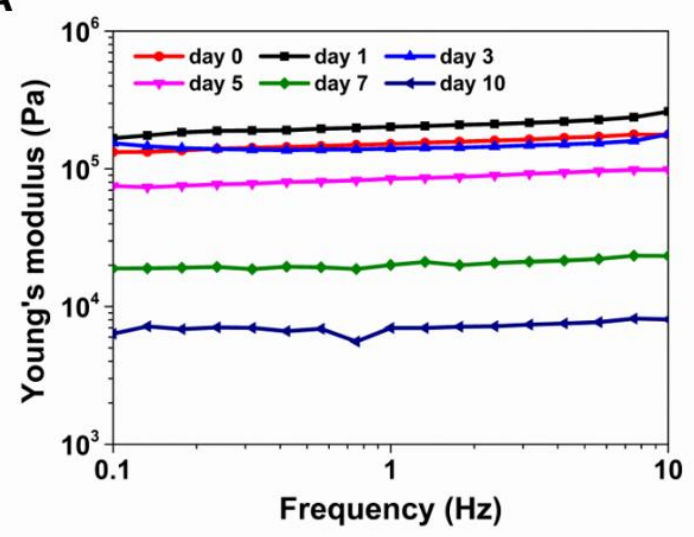

B

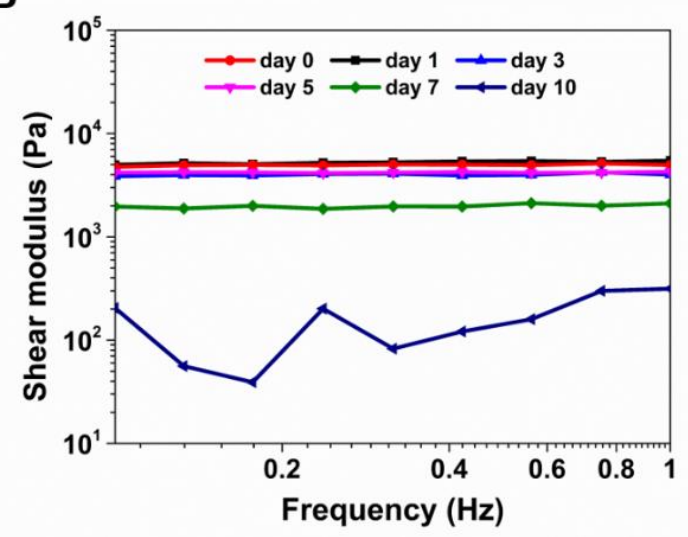

Figure S1. Mechanical properties of the GelMA/Gel hydrogels. Changes in (A) Young's modulus and (B) shear modulus of the GelMA/Gel hydrogels at different incubation periods in culture medium.

A

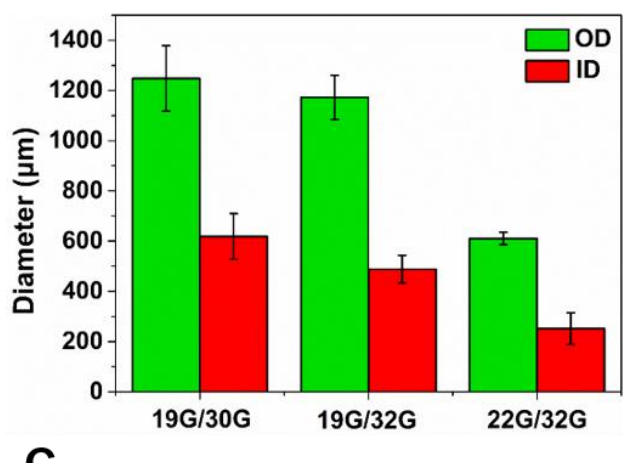

C

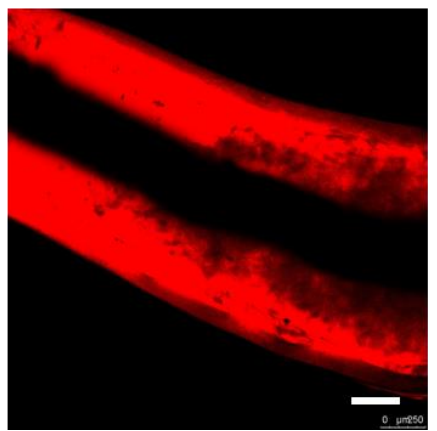

B

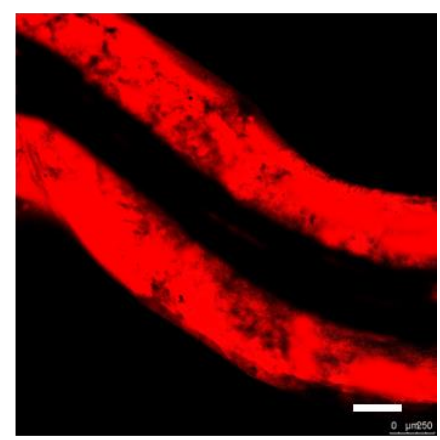

D

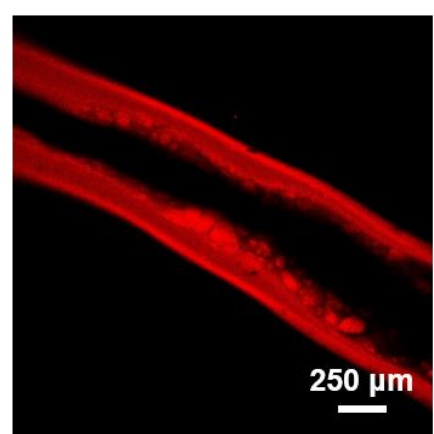

Figure S2. GelMA/Gel hollow microfibers fabricated at different gauge combinations of the needles. (A) ODs and IDs of the hollow microfibers fabricated at the different gauge combinations of the co-axial nozzles. (B-D) CLSM images of longitudinal cross-sections of the hollow microfibers corresponding to the different gauge combinations of the co-axial nozzles shown in (A). 
Table S1. Processing variables used for the preparation of hollow microfibers fabricated with various gauge combinations of the co-axial nozzles.

\begin{tabular}{|c|c|c|c|c|c|}
\hline Nozzle size & $\begin{array}{c}\text { Sample flow rate } \\
\qquad(\mu \mathrm{L} / \mathrm{min})\end{array}$ & $\begin{array}{c}\text { Core flow rate } \\
\qquad(\mu \mathrm{L} / \mathrm{min})\end{array}$ & $\begin{array}{l}\text { OD } \\
(\mu \mathrm{m})\end{array}$ & $\begin{array}{c}\text { ID } \\
(\mu \mathrm{m})\end{array}$ & $\begin{array}{c}\text { Wall thickness }^{a} \\
(\mu \mathrm{m})\end{array}$ \\
\hline $19 \mathrm{G} / 30 \mathrm{G}$ & 450 & 40 & 1248.1 & 618.4 & 314.9 \\
\hline $19 \mathrm{G} / 32 \mathrm{G}$ & 450 & 40 & 1172.2 & 479.9 & 346.2 \\
\hline $22 \mathrm{G} / 30 \mathrm{G}$ & 300 & 40 & 610.2 & 251.4 & 179.4 \\
\hline
\end{tabular}

${ }^{a}$ Wall thickness $=(O D-I D) / 2$ 